'Departamento de Medicina, Sección de Medicina Nuclear, Hospital Clínico Universidad de Chile. Santiago, Chile.

${ }^{2}$ Servicio de Radiología e Imágenes, Unidad de Medicina Nuclear. Clínica Santa María. Santiago, Chile.

aprograma de formación de especialista en Medicina Nuclear. Escuela de Postgrado. Facultad de Medicina, Universidad de Chile. Santiago, Chile. ${ }^{\mathrm{b}}$ Tecnólogo Médico.

Fuentes de apoyo financiero: No.

Recibido el 16 de febrero de 2018, aceptado el 13 de septiembre de 2018

Correspondencia a:

Dr. Patricio González Espinoza Avenida Santa María 0500,

$1^{\circ}$ Piso, Providencia. Santiago, Chile. pgonzalez@clinicasantamaria.cl

\section{Tratamiento con radioyodo endovenoso y estimulación con TSH recombinante humana en paciente con cáncer de tiroides extenso. Caso clínico}

\author{
JANE SPULER ${ }^{1, a}$, GABRIELA PAILLAHUEQUE ${ }^{1, a}$, \\ PATRICIO GONZÁLEZ ${ }^{1,2}$, DAVID CAMPOS ${ }^{2, \mathrm{~b}}$
}

\section{Intravenous radioiodine and recombinant human TSH in a patient with extensive thyroid cáncer. Report of one case}

We report a 72-years-old male patient with extensive differentiated thyroid cancer (DTC), who required a tracheostomy and gastrostomy. Considering his clinical condition, risk of aspiration and management of the ostomies, radioiodine $\left(131^{I}\right)$ was administered intravenously, using recombinant human thyrotropin (rhTSH) and levothyroxine. The procedure was successful, both clinically and in terms of radioprotection.

(Rev Med Chile 2018; 146: 1220-1223)

Key words: Gastrostomy; Iodine Radioisotopes; Tracheostomy; Thyrotropin Alfa; Thyroid Neoplasms.
$\mathrm{E}$ 1 tratamiento con radioyodo $\left({ }^{131} \mathrm{I}\right)$ está indicado en pacientes seleccionados con cáncer diferenciado de tiroides (CDT), ya sea como terapia ablativa, tratamiento de tumor no resecable o resección incompleta de tumor ${ }^{1}$. La administración del ${ }^{131}$ I usualmente es vía oral, en cápsulas o líquido.

\section{Objetivo}

Presentar tratamiento endovenoso de ${ }^{131}$ I en un paciente adulto mayor, complejo, con CDT extenso operado, con traqueostomía y gastrostomía y estimulación mediante tirotropina recombinante humana (TSHrh), con el cual se logra un manejo completamente satisfactorio desde el punto de vista clínico y de radioprotección. La administración endovenosa es un procedimiento infrecuente y con escaso reporte en la literatura ${ }^{2}$. No se encontró reporte de ${ }^{131}$ I endovenoso junto a TSHrh.

\section{Caso clínico}

Hombre de 72 años, proveniente del sur del país, con antecedentes de diabetes mellitus tipo 2 no insulino-requirente, hipertensión arterial, demencia leve y síndrome de apnea obstructiva del sueño, usuario de CPAP (Continuous Positive Airway Pressure), a quien se le diagnosticó CDT, con metástasis ganglionar cervical. El 06/01/2015 se realizó tiroidectomía total más disección bilateral de cuello.

Debido a la extensión del tumor, presentó parálisis bilateral cordal y trastorno de deglución, requiriendo traqueostomía para manejo de la vía aérea y gastrostomía para alimentación. La biopsia informó carcinoma papilar, variedad esclerosante difusa, con extenso compromiso extraglandular, invasión de la glándula paratiroidea incluida en las muestras, numerosas permeaciones tumora- 
les vasculares linfáticas y compromiso del borde quirúrgico, así como extensas metástasis intra y perinodales en los grupos II, III, IV, V y VII bilaterales (29 de 40 ganglios comprometidos), de hasta $3,1 \mathrm{~cm}$ de diámetro mayor. Se realizó tomografía computada de tórax que no demostró metástasis pulmonares.

Fue referido por su endocrinólogo como un T3N1bM0, según TNM (AJCC, 2010) ${ }^{3}$, quien indicó la administración de $5.550 \mathrm{MBq}(150 \mathrm{mCi})$ de ${ }^{131} \mathrm{I}$, por corresponder a un paciente con alto riesgo de recurrencia, según guías ATA $2015^{4}$, mediante la estimulación con TSHrh (administración intramuscular de $0,9 \mathrm{mg}$ a las 48 y $24 \mathrm{~h}$ antes del radioyodo) y así mantener la ingesta de $150 \mathrm{mcg}$ de levotiroxina, esto último con el fin de prevenir la morbilidad asociada a la suspensión de hormona tiroidea 5 . Considerando el antecedente de demencia leve, trastorno de la deglución y el manejo de sus dos ostomías, se decidió tratamiento con radioyodo endovenoso. En la evaluación inicial se corroboró manejo adecuado de esfínteres (especialmente vesical).

El paciente fue hospitalizado en habitación con blindaje de radioprotección. El mismo día del tratamiento se midió el nivel de TSH posterior a la TSHrh, que fue de $75 \mathrm{uU} / \mathrm{ml}$. Para administrar la dosis de radioyodo, y a modo de aumentar la distancia respecto del paciente y facilitar las condiciones de protección radiológica, se utilizaron alargadores conectados al acceso venoso del paciente que permitieron trabajar sobre superficie cubierta con biombo plomado de $2,5 \mathrm{~cm}$ de espesor. Durante la administración, el personal se dispuso detrás de biombo plomado, trabajando con las manos por sobre la barrera descrita e inyectando el ${ }^{131} \mathrm{I}$ líquido mediante jeringa cubierta con elemento plomado. Las dosis recibida por el personal expuesto durante la administración fue de $0,1 \mathrm{mSv}$.

Además, se administró suero glucosalino (2 L/ día) para facilitar la eliminación del ${ }^{131}$ I residual por vía urinaria y disminuir la exposición a radiación, no afectando la captación en el tejido tiroideo. (Zhindon JP, Carmona J, González P. Reducción de tasa de exposición a radiación en radioyodoterapia con utilización de líquidos endovenosos en cáncer diferenciado de tiroides. Estudio de 20 pacientes con y sin uso de líquidos endovenosos. Observaciones para enviar a publicación). El paciente se alimentó exclusivamente por gastrostomía durante su hospitalización.

El proceso se completó, como es habitual, con el rastreo de cuerpo completo, realizado al tercer día postratamiento $y$, además, un control al undécimo día. Estas imágenes mostraron múltiples metástasis en adenopatías cervicales, también presencia de captación intestinal y en los bordes de la traqueostomía y de la gastrostomía, como está descrito clásicamente para ${ }^{131} \mathrm{I}$ en procesos inflamatorios inespecíficos y por eliminación del radiofármaco por las mucosas ${ }^{2,6,7}$ (Figura 1: a, b yc).

El paciente firmó consentimiento informado como parte del protocolo clínico del Comité de Ética del establecimiento.

\section{Discusión}

La forma convencional de realizar tratamiento con ${ }^{131}$ I para hipertiroidismo y CDT es la vía oral, ya sea cápsula o líquido. En el caso de pacientes con CDT tiroidectomizados, la TSH se eleva al no administrar hormona tiroidea.

En el caso presentado, se decidió realizarlo vía endovenosa y por indicación de su endocrinólogo tratante, manteniendo la ingesta de hormona tiroidea por las siguientes razones: primero, por su condición global y deterioro cognitivo, segundo, por la dificultad de deglutir y el riesgo de aspiración $^{8}$ de radioyodo hacia la vía aérea y tercero, porque se estimó que ya sea una solución líquida o cápsula administrada por la gastrostomía podría conducir a situaciones complejas de manejo de la radioprotección. Afortunadamente, el paciente tenía buen control del esfínter urinario.

Si bien realizar dosimetría interna habría sido de interés, en la práctica ha demostrado ser compleja y costosa, por lo que no se realizó.

La vía venosa facilitó enormemente el manejo inicial, ya que aseguraba no tener grandes actividades del radioyodo en el estómago, salvo la captación y eliminación fisiológica luego de la administración parenteral.

Un beneficio adicional fue que el paciente no presentó ninguna sintomatología gastrointestinal adversa (náuseas, vómitos) por el radiofármaco, al evitar el tránsito inicial por estómago.

La administración de solución glucosalina, para aumentar la diuresis y disminuir la exposición a radiación, no modifica la tasa de exposi- 


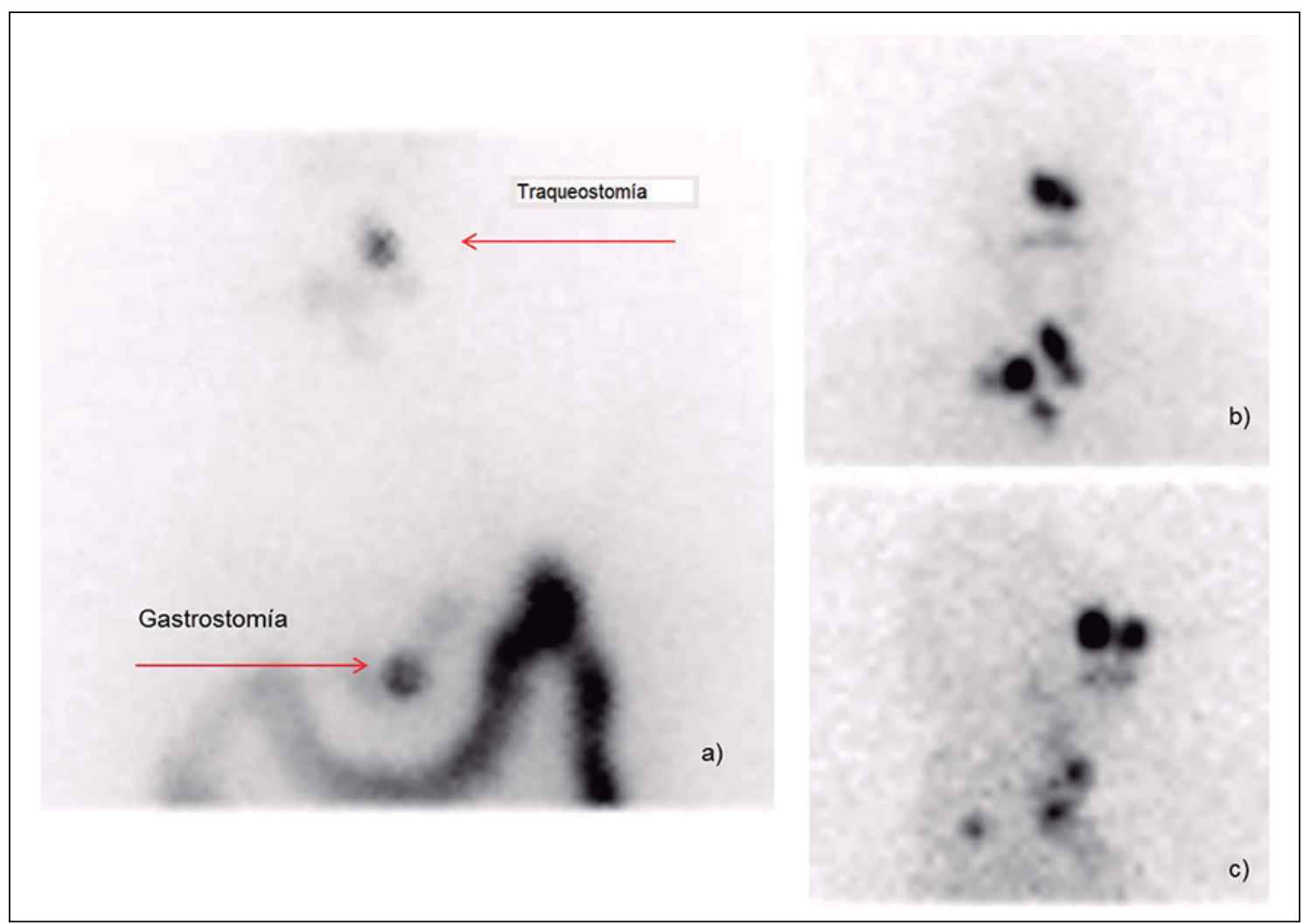

Figura 1. Rastreo de cuerpo entero: a) Proyección anterior cervical, tórax y abdomen al 3er día: Captación en región cervical en relación a borde de la traqueostomía; en abdomen: captación intestinal y en gastrostomía (flechas rojas). b) y c) Proyección cervical anterior y lateral respectivamente al $11^{\circ}$ día: captación en linfonodos metastásicos cervicales, no siendo visible la captación en traqueostomía por limpiado tardío.

ción en las primeras 24 h; está demostrado que la absorción del ${ }^{131} \mathrm{I}$ y su fijación se produce en las primeras 6 horas, por lo que no se arriesga menor efectividad ${ }^{9}$ (Zhindon JP, Carmona J, González P. Reducción de tasa de exposición a radiación en radioyodoterapia con utilización de líquidos endovenosos en cáncer diferenciado de tiroides. Estudio de 20 pacientes con y sin uso de líquidos endovenosos. Observaciones para enviar a publicación).

A 2 años del tratamiento, el paciente no ha presentado recidiva y se mantiene en buenas condiciones clínicas, en control endocrinológico.

El adecuado efecto obtenido en este caso apoya la vía endovenosa como excelente alternativa en casos complejos como el presentado, incluyendo pacientes con traqueostomía y gastrostomía.

Como desventaja se menciona que el costo del ${ }^{131}$ I para uso endovenoso es más alto que la formulación oral.

La indicación del tratante de usar TSHrh se justifica para prevenir una descompensación de su cuadro general, especialmente cognitivo, secundaria al hipotiroidismo. Está demostrado que la efectividad de la TSHrh es similar a la suspensión de hormona en pacientes sin evidencia de metástasis a distancia que tienen un bajo, intermedio $o$ alto riesgo de recurrencia ${ }^{10}$. Asimismo, se ha estudiado su uso pacientes con metástasis a distancia con similares tasas de sobrevivida global a 5 años que en aquellos en los cuales se preparan mediante suspensión de la hormona tiroidea ${ }^{11}$. Sin embargo, aún faltan estudios que aseguren que las dosis absorbidas por las lesiones son similares con preparación con TSHrh versus suspensión de hormona tiroidea ${ }^{12}$. 
En la literatura reciente se encontró el caso de un paciente con gastrostomía y administración endovenosa, sin $\mathrm{TSHrh}^{2}$. Los autores se concentraron en la cinética cuantitativa del radioyodo en el abdomen. El paciente presentado en nuestra publicación es clínicamente más complejo y con imágenes que fueron suficientes para conocer la distribución del radioyodo, destacando la captación intestinal y, en menor grado, del estómago. Además, este sería el primer reporte de ${ }^{131} \mathrm{I}$ endovenoso y TSHrh.

Otro estudio compara el uso de ${ }^{131}$ I oral con la vía endovenosa para el tratamiento del hipertiroidismo, no mostrando diferencia en las dosis efectivas $^{13}$. Si bien, no es patología maligna, se puede extrapolar que el comportamiento del radioyodo debiera ser similar al caso clínico presentado en que se usó la vía endovenosa.

\section{Conclusión}

En pacientes complejos como el presentado en este caso, la administración endovenosa de ${ }^{131}$ I junto a TSHrh, sin suspensión de ingesta de hormona tiroidea, es una excelente alternativa a la vía oral, siendo segura desde el punto de vista de protección radiológica.

\section{Referencias}

1. Luster M, Clarke SE, Dietlein M, Lassmann M, Lind $\mathrm{P}$, Oyen WJ, et al. Guidelines for radioiodine therapy of differentiated thyroid cancer. Eur J Nucl Med Mol Imaging 2008; 35: 1941-59.

2. Scuffham JW, Wood KA, Clauss RP, Kilfeather SA, Hinton PJ. Radioiodine retention on percutaneous endoscopic gastrostomy tubes. Br J Radiol 2012; 85 (1012): e076-e078.

3. Edge SB, Byrd DR, Compton CC, Fritz AG, Greene FL, Trotti A (eds). AJCC Cancer Staging Manual. 7th edition. Springer-Verlag, New York, 2009. pp 59-64.

4. Haugen BR, Alexander EK, Bible KC, Doherty GM, Mandel SJ, Nikiforov YE, et al. 2015 American Thyroid Association Management Guidelines for Adult Patients with Thyroid Nodules and Differentiated Thyroid Can- cer: The American Thyroid Association Guidelines Task Force on Thyroid Nodules and Differentiated Thyroid Cancer. Thyroid 2016; 26 (1): 1-133.

5. Luster M, Felbinger R, Dietlein M, Reiners C.Thyroid hormone withdrawal in patients with differentiated thyroid carcinoma: a one hundred thirty-patient pilot survey on consequences of hypothyroidism and a pharmacoeconomic comparison to recombinant thyrotropin administration. Thyroid 2005; 15: 1147-55.

6. Oh JR, Ahn BC. False-positive uptake on radioiodine whole-body scintigraphy: Physiologic and pathologic variants unrelated to thyroid cancer. Am J Nucl Med Mol Imaging 2012; 2: 362-85.

7. Rudoni S, Toubeau M, Mansuy S, Vaillant G, Verges $\mathrm{B}$, Brun JM, et al. False positive scintigraphic images in the surveillance of differentiated thyroid cancers. Ann Endocrinol 1997; 58: 399-407.

8. Elpern EH, Borkgren Okonek M, Bacon M, Gerstung C, Skrzynski M. Effect of the Passy Muir tracheostomy speaking valve on pulmonary aspiration in adults. Heart Lung 2000; 29: 287-93.

9. National Council on Radiation Protection and Measurements. Dose limits for individuals who receive exposure from radionuclide therapy patients. NCRP Commentary Number II. Bethesda, MD: NCRP; 1995.

10. Sabra M, Tuttle RM. Recombinant human thyroid-stimulating hormone to stimulate 131-I uptake for remnant ablation and adjuvant therapy. Endocrine Prac 2013; 19: 149-56.

11. Tala H, Robbins R, Fagin JA, Larson SM, Tuttle RM. Five-year survival is similar in thyroid cancer patients with distant metastases prepared for radioactive iodine therapy with either thyroid hormone withdrawal or recombinant human TSH. J Clin Endocrinol Metabol. 2011; 96 (7): 2105-11.

12. Plyku D, Hobbs RF, Huang K, Atkins F, Garcia C, Sgouros G, et al. Recombinant human thyroid-stimulating hormone versus thyroid hormone withdrawal in 124I-PET/CT based dosimetry for 131I therapy of metastatic differentiated thyroid cancer. J Nucl Med 2017; 58: 1146-54.

13. Schneider P, Biko J, Hänscheid H, Hilliger S, Koutsampelas C, Kranzfelder M, et al. The route of administration (oral vs intravenous) does not influence dose or outcome in Graves' disease and unifocal autonomy. Eur J Nucl Med Mol Imaging 2005; 32: 788-93. 